\title{
Representative Sample Sampling Method for Size Effect Experiment of Jointed Rock Mass
}

\author{
Man Huang $\left(\mathbb{D},{ }^{1}\right.$ Dan Liu, ${ }^{1}$ Chenjie Hong $\left(D,{ }^{1}\right.$ Shigui Du, ${ }^{1}$ Zhanyou Luo, ${ }^{2}$ Changhong Li, ${ }^{3}$ \\ and Yongliang Huang 4 \\ ${ }^{1}$ Department of Civil Engineering, Shaoxing University, 508 Huancheng West Road, Shaoxing 312000, China \\ ${ }^{2}$ Institute of Geotechnical Engineering, Zhejiang University of Science and Technology, 508 Liuhe Road, Hangzhou 310023, China \\ ${ }^{3}$ Zhejiang Geological Prospecting Industry Group Co. LTD., 160 Renmin Zhong Lu, Shaoxing 312000, China \\ ${ }^{4}$ Zhejiang Bureau of Nonferrous Metal Geological Exploration, 160 Renmin Zhong Lu, Shaoxing 312000, China
}

Correspondence should be addressed to Chenjie Hong; hongchenjie66@163.com

Received 16 July 2020; Revised 6 August 2020; Accepted 11 August 2020; Published 25 August 2020

Academic Editor: Hang Lin

Copyright (c) 2020 Man Huang et al. This is an open access article distributed under the Creative Commons Attribution License, which permits unrestricted use, distribution, and reproduction in any medium, provided the original work is properly cited.

The influence of size effect on the hydromechanical behavior of rock mass has long been recognized. As a result, analysis of the rock mass size effect has been developed. However, when conducting size effect studies, the representativeness of the sample is less considered. Therefore, combining the existing statistical methods and sampling methods, a comprehensive representative sampling method for rock mass is present. In this method, a large number of sample statistics of different sizes are provided in the progressive coverage method; then, the plane density of the track length is defined as the value of stratification to perform stratified sampling for representative samples. Furthermore, it is applied to a joint network model generated in accordance with the actual situation and compared with those of other methods. The sampling results show that the proposed method can provide a certain reference value for studying the scale-dependent behavior of rock mass mechanical parameters.

\section{Introduction}

The mechanical properties of rock mass have many applications in engineering practices, such as tunnels, dams, and slopes; the influence of size effect on it is investigated $[1,2]$. That is, the mechanical parameters are primarily attributed to the variation in geometric dimensions of the specimen [3-7]. In engineering practice, the mechanical parameters of large-scale specimens are often obtained by reducing the test results of small-scale mechanical parameters, but the reliability of the results is still difficult to clarify. Therefore, an in-depth study of the scaledependent behavior of mechanical properties of rock mass enables the mechanical parameters to be determined accurately, thereby offering certain guiding significance for the application of parameters in engineering practice.

The study on the size effect of rock mass mechanical properties has been conducted through laboratory tests, numerical simulation, in situ tests, etc. [8-10]. The mechan- ical test mainly includes compression, shear, and tension, by performing laboratory tests that can only obtain the strength of the intact rock being restricted to indoor conditions. However, the strength should be decreased for application in practical engineering [11-13]. Subsequently, various numerical simulation software, including finite element and discrete element software, has been adopted by more authors. These software can simulate the mechanical test of real rock mass, customize the strength level of rock mass, and set different types of mechanical tests [14-17]. In addition, the anisotropic properties of the rock mass were further studied [18, 19]. Few researchers conducted field investigations through in situ tests, because these methods are expensive and impractical [20-24]. Therefore, among the above methods, the numerical simulation can approximately meet test requirements under various conditions and also repeatedly perform numerical calculations to reduce the error of the test results, thus serving as a feasible research method. 


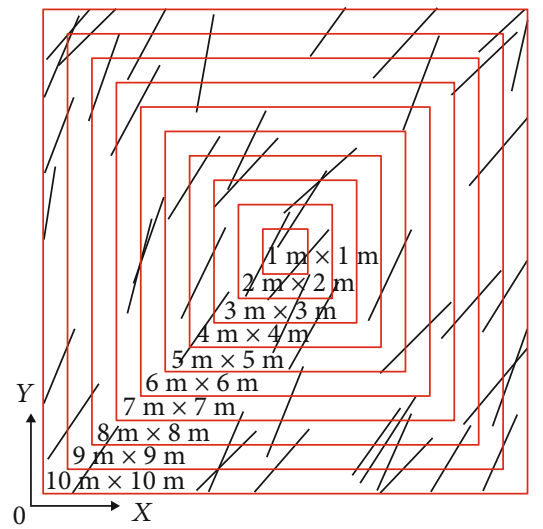

(a)

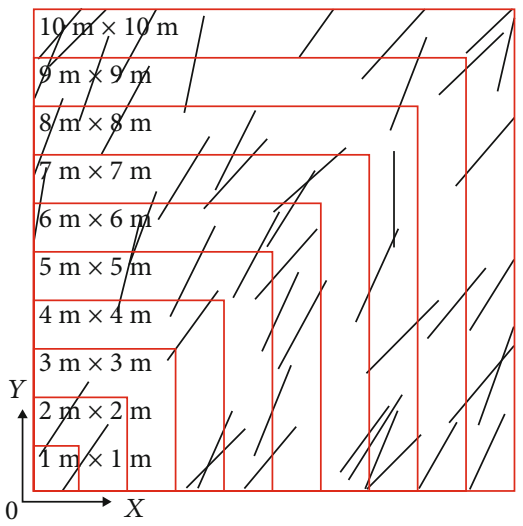

(b)

FIGURE 1: Processive magnifying sampling method: (a) middle amplification; (b) side amplification.

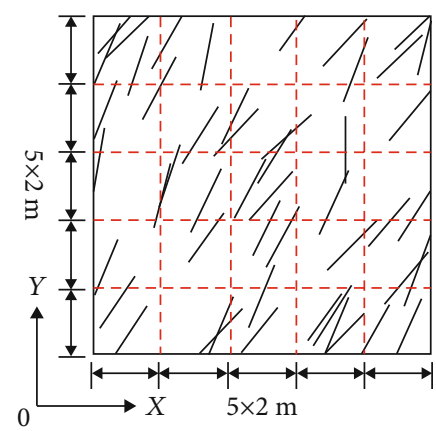

FIGURE 2: Equal-partition sampling method.

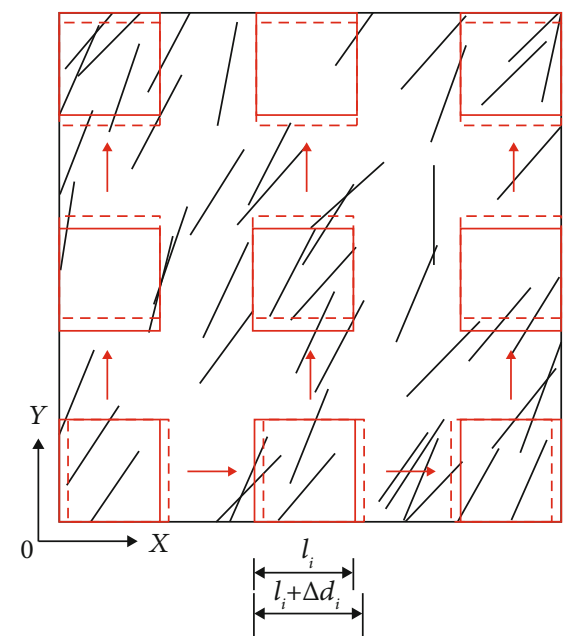

FIgURE 3: Progressive coverage statistical method.

The reasonable selection of samples on the basis of statistical samples is essential prior to numerical simulation. Regarding the study on the size effect law, it is required that large-size samples include small-size samples to acquire the correct size effect law. However, current research has been mainly based on a single sample and a random sample [18, 25-27]. The obtained size effect laws differ and show that the sample value increases (positive size effect), decreases (negative size effect), and remains constant (no size effect), among other results, as a function of the sampling size. However, the representativeness of the samples obtained by this method has not yet been determined; therefore, the reliability of the results is questionable. Representative sampling methods have been advanced to address the above problems. Such methods include stratified sampling, which was well applied in rock joints. However, the feasibility of jointed rock mass needs to be further explored and analyzed. Therefore, a method based on the joint network model and combined with the progressive coverage method for sample statistics and stratified sampling is needed for representative sampling.

In this study, a comprehensive rock sample sampling method is suggested and applied to the acquisition of series-sized rock samples. Furthermore, the rationality of the sampling results obtained by the proposed method is tested with the joint network model, and its advantages and roles are compared with those of the other sampling methods. This step verifies if the scale-dependent behavior of mechanical properties is effective.

\section{Compare and Analyze Different Sampling Methods}

\subsection{Single-Sample Sampling Method}

2.1.1. Simple Random Sampling Method. As a result of the complexity and variability of rock mass structure in the field, different-size samples are generally arbitrarily taken from the original rock mass, and the choice of sample positions primarily depends on individual subjective judgments $[18,27]$.

2.1.2. Processive Magnifying Sampling Method. The processive magnifying sampling method refers to the selection of the starting point for sampling within the scope of the size; this point is used as the sampling reference every time and is gradually expanded to obtain rock samples of various sizes in turn $[25,26]$. For example, the left bottom or the center is utilized as the starting point to gradually expand (Figure 1). This method can overcome the irregularity of the random sampling method, but the representativeness of the obtained sample is still unclear. 


\subsection{Multisample Sampling Method}

2.2.1. Equal-Partition Sampling Method. This method can be applied to divide an entire large rock mass at equal intervals, as shown in Figure 2, to obtain original samples at different locations. A large sampling size corresponds to a small sample capacity. Therefore, the method may not provide a suitable sample capacity or guarantee that a complete set of continuously sized samples will be provided when full coverage is achieved.

2.2.2. Progressive Coverage Statistical Method. To provide a comprehensive sampling basis for the series-size sample statistics, Huang et al. proposed the progressive coverage statistical method, which is an effective tool for seriessize sample statistics [28]. The key is to determine the sampling size $l_{i}$ and the propulsion space $\Delta d_{i}$ ( $i$ is the corresponding number of different sizes). The sampling unit gradually advances along the $x$ and $y$ directions of the rock mass, and then, the samples achieve coverage; a schematic diagram is given to reflect in Figure 3. This method obtains more complete samples, which can cover some missing areas, increase the sample capacity, improve the accuracy of the overall samples, and provide a certain sampling basis.

2.3. Representative Sampling Method. The stratified sampling method was raised to improve the situation of an excessive number of small samples [29]. This method was originally applied to rock joints. At present, in using this method to the sampling of rock mass, the basic parameter of stratification is defined first. Then, the parameter is arranged from small to large, and the stratification ratio is determined by using the quartile method of this parameter. All the statistical values are divided into three intervals, namely, 0-25\%, 25-75\%, and 75$100 \%$, where the distribution proportion of samples in each layer is $1 / 4,1 / 2$, and $1 / 4$, respectively. The sample capacity is counted within the allowable error range. Then, representative samples in the corresponding layers are selected, and the sampling capacity is minimized, thus enabling them to be reasonably distributed. Subsequently, the stratified sampling method is further improved, and a new situation is added to the sample layer. When the relative range of the basic parameters is less than $10 \%$, an interval of $0 \%-100 \%$ is defined for the statistical values, and the distribution ratio is 1 .

In summary, a comprehensive rock mass sampling method that combines statistical and sampling methods has not yet been proposed. Therefore, a new sampling method needs to be established to ensure an equal sample capacity of different sampling sizes, and the representative samples are chosen from them, which provide a theoretical basis for studying the scale dependency of mechanical properties of rock mass on size.

\section{Representative Sampling Method Based on a Joint Network Model}

On the basis of a comparative analysis of different methods, a representative sampling method for the joint network model is advanced in this paper. Its detailed steps are described below.

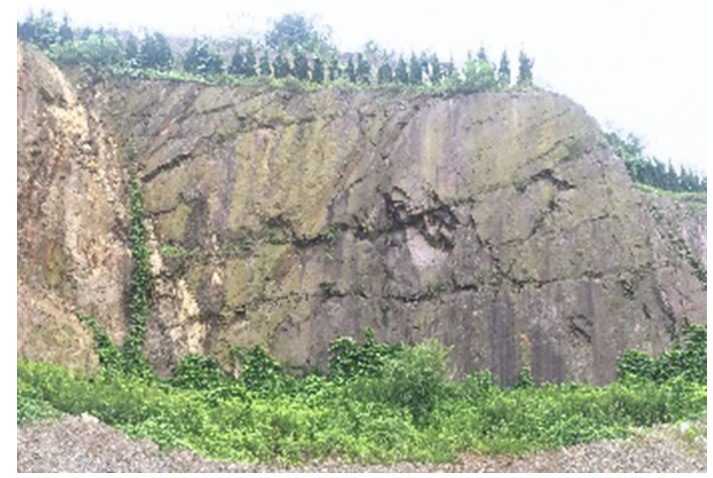

FIgUre 4: Mine slope in Shaoxing, China.

3.1. Establishment of a Joint Network Model Based on a Jointed Rock Mass. Statistical analysis of joint parameters provides basic data for the joint network model by collecting parameter information, such as trace length, spacing, and dip angle of the rock mass in the field. In generating the joint network model, the size of the simulation area is set first, that is, the rock mass with a dimension of $L \times L$, and the distribution form of joint trace length, spacing, and dip angle is determined. Then, the joint center point coordinates $\left(x_{0}, y_{0}\right)$, trace length $l_{t}$, and dip angle $\theta$ are converted into joint end point coordinates $A$ $\left(x_{1}, y_{1}\right)$ and $B\left(x_{2}, y_{2}\right)$ according to Formula (1). If any end point is not in the simulation area, then the intersection point of the joint trace and the area boundary is taken as the joint end point. The joint network model diagram is provided through software programming.

$$
\begin{aligned}
& x_{1,2}=x_{0} \pm \frac{l_{t}}{2} \cos \theta, \\
& y_{1,2}=y_{0} \pm \frac{l_{t}}{2} \sin \theta .
\end{aligned}
$$

3.2. Series-Size Sample Statistics. The progressive covering method is employed to set square sampling units of different sizes and the propulsion space and then gradually advance along the orthogonal direction of the rock mass to achieve appropriate sampling capacity. The calculation formula of sample capacity $N$ is

$$
N=\left[\frac{L-l_{i}}{\Delta d_{i}}+1\right]^{2}
$$

3.3. Representative Sampling of the Single Size. The plane density of the trace length is one of the important parameters that characterize the internal geometric distribution of the rock mass, which mainly represents the influence of the joint trace length and spacing on the rock mass. The parameter will have a certain influence on the mechanical properties of the rock mass. For this purpose, the plane density of the track length is taken as the basic parameter for layering. This parameter is defined as the length of all joints contained in the unit area of the rock mass [30]. The calculation 
TABLE 1: Distribution statistics of joint geometric parameters.

\begin{tabular}{lcccccccc}
\hline \multirow{2}{*}{ Joint } & \multicolumn{2}{c}{ Spacing $(\mathrm{m})$} & \multicolumn{2}{c}{ Trace length $(\mathrm{m})$} & \multicolumn{3}{c}{ Dip angle $\left(^{\circ}\right)$} & \multicolumn{2}{c}{ Variance } \\
& Distribution & Mean & Distribution & Mean & Variance & Distribution & Mean & Variance \\
\hline 1 & Negative exponential & 1.02 & Normal & 2.47 & 0.12 & Normal & 57.8 & 8.8 \\
2 & Negative exponential & 0.98 & Normal & 1.97 & 0.14 & Normal & 60.3 & 9.2 \\
3 & Negative exponential & 1.05 & Normal & 1.88 & 0.09 & Normal & 29.4 & 10.1 \\
\hline
\end{tabular}

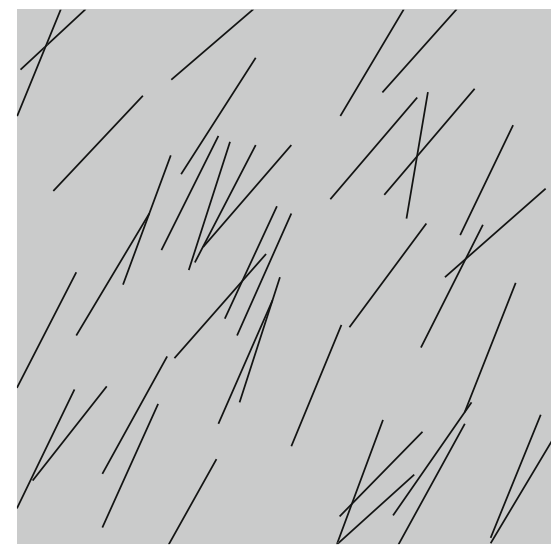

(a)

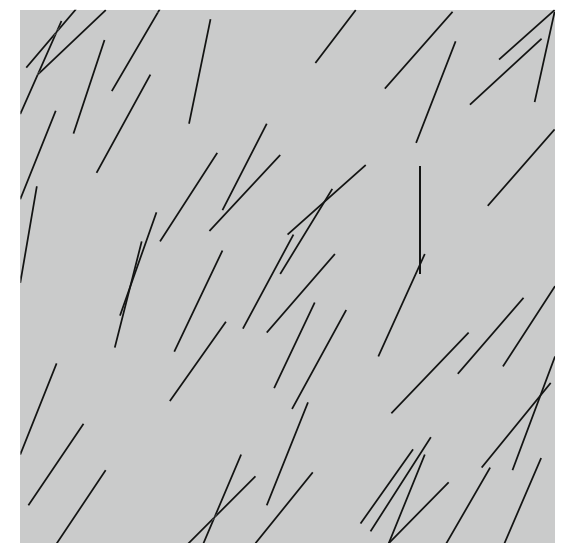

(b)

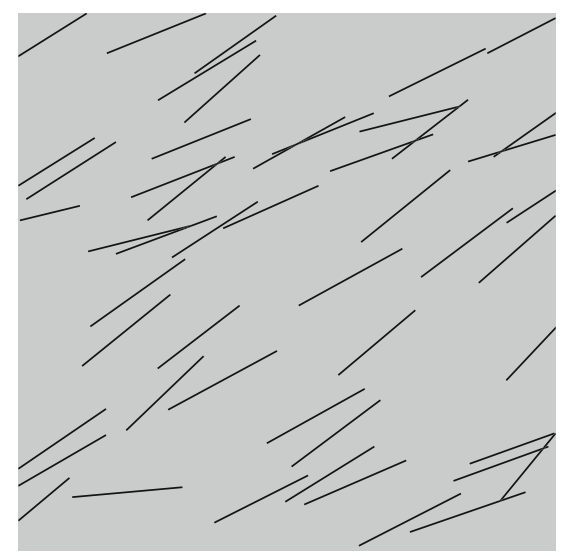

(c)

Figure 5: Joint network model of different groups: S1; S2; S3.

TABle 2: Sample capacity statistics for different sampling sizes.

\begin{tabular}{lccccc}
\hline Sampling size $(\mathrm{m} \times \mathrm{m})$ & Propulsion space $(\mathrm{m})$ & Sample capacity & Sampling size $(\mathrm{m} \times \mathrm{m})$ & Propulsion space $(\mathrm{m})$ & Sample capacity \\
\hline $1 \times 1$ & 0.9 & 121 & $6 \times 6$ & 0.4 & 121 \\
$2 \times 2$ & 0.8 & 121 & $7 \times 7$ & 0.3 & 0.2 \\
$3 \times 3$ & 0.7 & 121 & $9 \times 8$ & 0.1 & 121 \\
$4 \times 4$ & 0.6 & 121 & $10 \times 10$ & 0 & 121 \\
$5 \times 5$ & 0.5 & 121 & & 1 & 1 \\
\hline
\end{tabular}

formula is as follows:

$$
L_{p}=\frac{\left(l_{t 1}+l_{t 2}+\cdots l_{t j}+\cdots+l_{t n}\right)}{l_{i} \times l_{i}},
$$

where $L_{p}$ is the plane density of the track length, $l_{t 1}, l_{t 2}, \cdots$ $, l_{t j}, \cdots, l_{t n}$ represent the length of each joint, and $j$ is the corresponding number for different joints. In this paper, the cumulative pixel points of the trace in the proportion of the study area are converted to the plane density of the trace 
TABLE 3: Information statistics of layered sampling with different sampling sizes.

\begin{tabular}{lccccc}
\hline Sampling size $(\mathrm{m} \times \mathrm{m})$ & The relative range & Sample capacity & Sampling size $(\mathrm{m} \times \mathrm{m})$ & The relative range & Sample capacity \\
\hline $1 \times 1$ & $399.10 \%$ & 16 & $6 \times 6$ & $36.31 \%$ & 4 \\
$2 \times 2$ & $252.77 \%$ & 8 & $7 \times 7$ & $8 \times 8$ & $24.98 \%$ \\
$3 \times 3$ & $148.86 \%$ & 4 & $9 \times 9$ & $8.17 \%$ & 4 \\
$4 \times 4$ & $103.05 \%$ & 4 & $10 \times 10$ & $0 \%$ & 1 \\
$5 \times 5$ & $56.17 \%$ & 4 & &
\end{tabular}

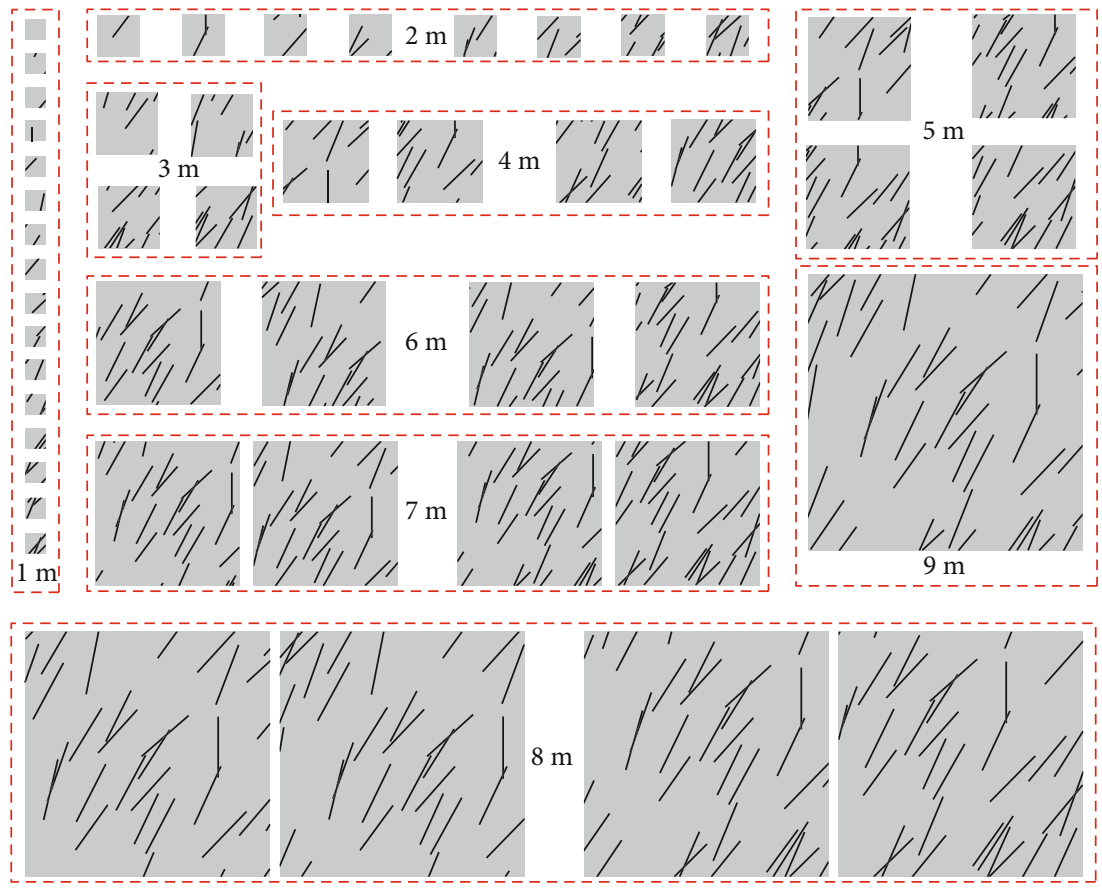

FIGURE 6: Sample distribution statistics of different size.

length, that is, the overall decay rate of $L_{p}$ will be a certain multiple, which is expressed as follows:

$$
L_{p m}=\frac{L_{p}}{m},
$$

where $m$ represents the pixels corresponding to unit length.

In accordance with the operational process of the stratified sampling method in Section 2.3, the minimum sample capacity that meets the requirements is calculated, the representative samples are ensured in the corresponding layers, and the sample distribution becomes more uniform.

The reliability and feasibility of the new sampling method are verified by comparing the sampling results with those of other methods.

\section{Application and Analysis}

4.1. Acquisition of Representative Series-Size Rock Samples. The internal joints of the rock mass are complex and changeable and cannot be considered one by one. Currently, the statistical distribution method is often employed to establish the model. The rock mass level model is established considering the distribution characteristics of rock mass macrocracks. After several field investigations, the research team collected data on three sets of rock mass joints in a mine slope in Shaoxing, China (Figure 4). The distributions of geometric parameters of joints are listed in Table 1. A single set of joints is the research object, and three sets of rock models with dimensions of $10 \mathrm{~m} \times 10 \mathrm{~m}$ are generated by software programming, as shown in Figure 5. The exported picture is uniformly adjusted to 300 pixels $\times 300$ pixels by using image processing technology.

Then, the progressive coverage method is suggested to fix 9 square sampling units of different sizes, with sampling sizes $l_{i}$ of $1 \mathrm{~m}, 2 \mathrm{~m}, \ldots, 9 \mathrm{~m}$, under the condition that the sample capacity of each size is 121 , and the propulsion space $\Delta d_{i}$ is $0.9 \mathrm{~m}, 0.8 \mathrm{~m}, \ldots, 0.1 \mathrm{~m}$ for rock sample acquisition. All sampling sizes can completely cover all areas. When the size is $10 \mathrm{~m}$, it is the original sample. The statistical information is presented in Table 2.

Many samples obtained by the progressive coverage method are representatively sampled in series size. The $m$ is set as 30. The statistical results are listed in Table 3. The findings show the relative range to decrease as a function of the sampling size, but when the size is $7 \mathrm{~m}$, a slightly increasing 


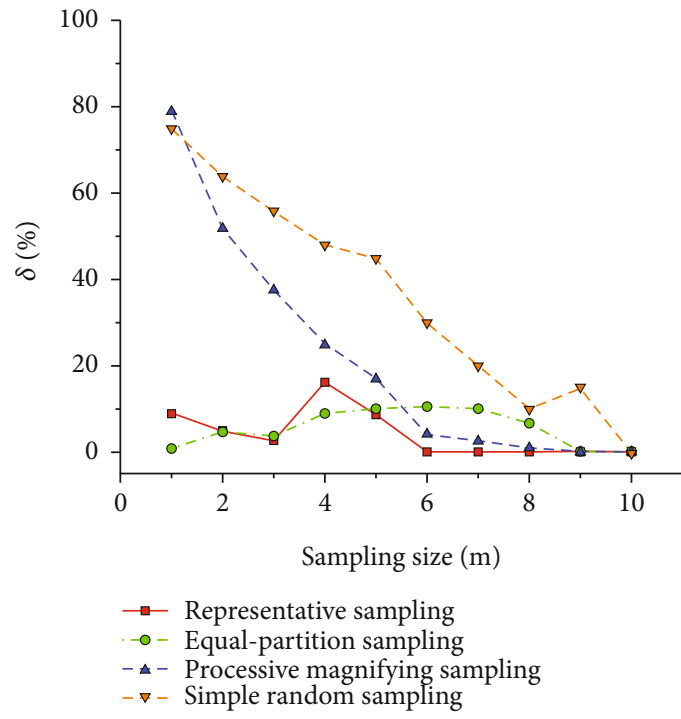

(a)

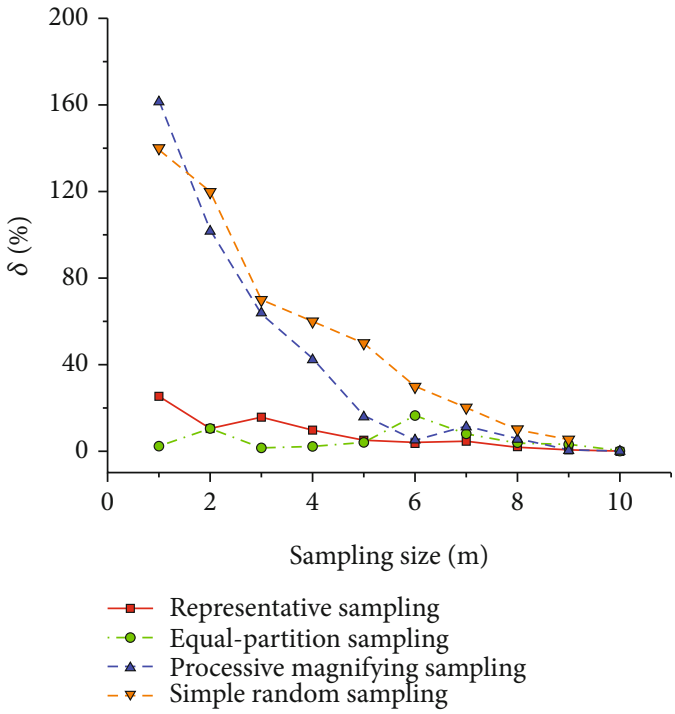

(b)

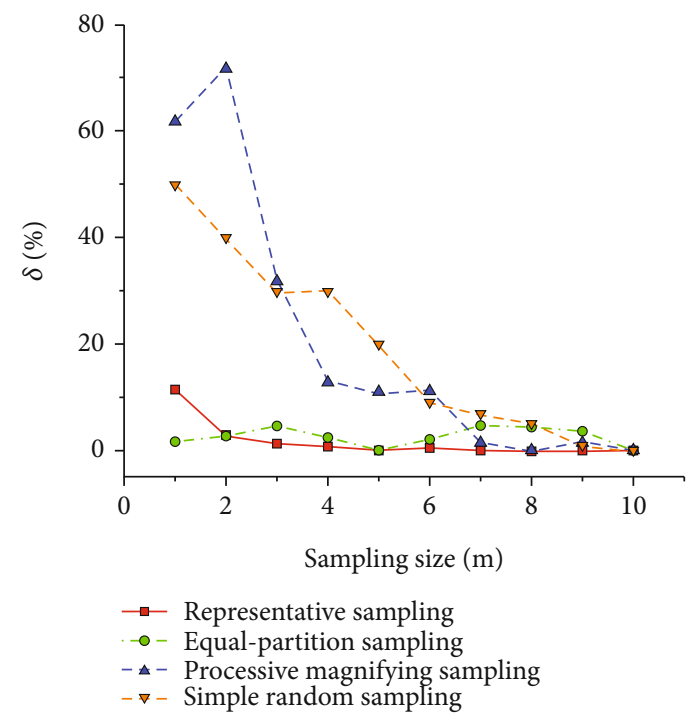

(c)

Figure 7: The relative error of different sampling methods applied in three sets of jointed rock masses: S1; S2; S3.

trend is observed. When the size is $6-8 \mathrm{~m}$, the sample capacity is 4 . When the size is $9 \mathrm{~m}$, the relative range is less than $10 \%$, and a sample is taken here. However, when the size is small, the sample capacity is large. A schematic of the distribution of series-size samples is taken, such as S2, as shown in Figure 6. The order of samples of each size increases from left to right and then from top to bottom.

4.2. Comparative Analysis of Sampling Results of Different Methods. The three joint network models of $10 \mathrm{~m} \times 10 \mathrm{~m}$ generated above are taken as examples to compare the sampling results of different methods. First, the model size varies from $1 \mathrm{~m}$ to $10 \mathrm{~m}$, and series-size samples are obtained through the representative sampling method, equalpartition sampling method, processive magnifying sampling method, simple random sampling method, and progressive coverage method. Second, the $L_{p}$ of each sample is calculated.
The sampling of the joint network model requires the selected samples to be able to represent the internal structure at the sampling size. Then, considering that the representative method is based on $L_{p}$, the $L_{p}$ of the sample obtained by the progressive covering method is the measured value, and the relative error between this method and other methods is established. The calculation formula is as follows:

$$
\delta=\frac{\left|R_{P}-R_{c}\right|}{R_{P}} \times 100 \%,
$$

where $R_{P}$ is the mean $L_{p}$ of the population and $R_{c}$ is the mean $L_{p}$ of the specimen.

As shown in Figure 7, for the application of different methods in different jointed rock mass, the results show that the overall relative error value of the processive magnifying 
sampling method and random sampling is greater than that of the other two methods. However, because of the randomness of the random sampling method, the relative error between the random sampling method and other methods is impossible to judge accurately. Except for individual sizes, the relative error of the equal-partition sampling method and the representative sampling method is less than $10 \%$. The results show that the relative errors of the two methods are small, but the former method has a large sample capacity when sampling in a small size, thereby increasing the complexity of the test operation. Therefore, the representative sampling method is the best choice for rock sample statistics. Here, based on representative samples, the compressive strength test is carried out, then the mechanical analysis of the sampling results is achieved, and the applicability of the method is further verified.

\section{Conclusion}

Considering the existing statistical and sampling methods, a comprehensive representative sampling method of rock mass is proposed, and its feasibility is verified with examples. The main conclusions are as follows:

(1) A new sampling method that comprises a progressive coverage method and stratified sampling method is proposed to minimize the sample capacity and make the distribution more reasonable. Numerous seriessize samples are adopted by the former, and the plane density of the track length is taken as the reference value for the latter to obtain representative samples

(2) The example analysis shows that this method can determine more representative samples in combination with the plane density of the track length on the basis of providing more sufficient samples, reduce the calculation workload, and provide a sampling basis for rock samples of various sizes. This method is of great significance in studying the size effect test law of rock mass mechanical properties

(3) An evaluation of the plane density of the track length of varying size obtained by different methods taken from the joint model reveals that the relative error of the representative sampling method is almost entirely below $10 \%$. Hence, the new sampling method is effective for rock mass sampling

\section{Data Availability}

The data used to support the findings of this study are included within the article.

\section{Conflicts of Interest}

The authors declare that there is no conflict of interest regarding the publication of this article.

\section{Acknowledgments}

This study has been partially funded by the Key Research and Development Projects of Zhejiang Province (No. 2019C03104), the Natural Science Foundation of Zhejiang Province (No. LY18D020003), and the National Natural Science Foundation of China (Nos. 41572299 and 41427802).

\section{References}

[1] D. Liu, Z. Gu, R. Liang et al., "Impacts of pore-throat system on fractal characterization of tight sandstones," Geofluids, vol. 2020, Article ID 4941501, 17 pages, 2020.

[2] Z. Tao, Y. Wang, C. Zhu, H. Xu, G. Li, and M. He, "Mechanical evolution of constant resistance and large deformation anchor cables and their application in landslide monitoring," Bulletin of Engineering Geology and the Environment, vol. 78, no. 7, pp. 4787-4803, 2019.

[3] C. Zhang, C. Pu, R. Cao, T. Jiang, and G. Huang, "The stability and roof-support optimization of roadways passing through unfavorable geological bodies using advanced detection and monitoring methods, among others, in the Sanmenxia Bauxite Mine in China's Henan Province," Bulletin of Engineering Geology and the Environment, vol. 78, no. 7, pp. 5087-5099, 2019.

[4] Y. Li, S. Sun, and C.'. Tang, “Analytical prediction of the shear behaviour of rock joints with quantified waviness and unevenness through wavelet analysis," Rock Mechanics and Rock Engineering, vol. 52, no. 10, pp. 3645-3657, 2019.

[5] M. Gao, Z. Liang, S. Jia, Y. Li, and X. Yang, "An equivalent anchoring method for anisotropic rock masses in underground tunnelling," Tunnelling and Underground Space Technology, vol. 85, pp. 294-306, 2019.

[6] N. Huang, R. Liu, Y. Jiang, Y. Cheng, and B. Li, "Shear-flow coupling characteristics of a three-dimensional discrete fracture network-fault model considering stress-induced aperture variations," Journal of Hydrology, vol. 571, pp. 416-424, 2019.

[7] J. Oh, T. Moon, I. Canbulat, and J. S. Moon, "Design of initial support required for excavation of underground cavern and shaft from numerical analysis," Geomechanics and Engineering, vol. 17, pp. 573-581, 2019.

[8] Y. Zhao, L. Zhang, W. Wang et al., "Creep behavior of intact and cracked limestone under multi-level loading and unloading cycles," Rock Mechanics and Rock Engineering, vol. 50, no. 6, pp. 1409-1424, 2017.

[9] R. Cao, P. Cao, X. Fan, X. Xiong, and H. Lin, “An experimental and numerical study on mechanical behavior of ubiquitousjoint brittle rock-like specimens under uniaxial compression," Rock Mechanics and Rock Engineering, vol. 49, no. 11, pp. 4319-4338, 2016.

[10] R. Cao, P. Cao, and H. Lin, "Mechanical behavior of brittle rock-like specimens with pre-existing fissures under uniaxial loading: experimental studies and particle mechanics approach," Rock Mechanics and Rock Engineering, vol. 49, pp. 763-783, 2016.

[11] A. A. Giwelli, K. Sakaguchi, and K. Matsuki, "Experimental study of the effect of fracture size on closure behavior of a tensile fracture under normal stress," International Journal of Rock Mechanics and Mining Sciences, vol. 46, no. 3, pp. 462470, 2009.

[12] W. J. Darlington, P. G. Ranjith, and S. K. Choi, “The effect of specimen size on strength and other properties in laboratory 
testing of rock and rock-like cementitious brittle materials," Rock Mechanics and Rock Engineering, vol. 44, no. 5, pp. 513-529, 2011.

[13] R. Yoshinaka, M. Osada, H. Park, T. Sasaki, and K. Sasaki, "Practical determination of mechanical design parameters of intact rock considering scale effect," Engineering Geology, vol. 96, no. 3-4, pp. 173-186, 2008.

[14] M. Bahaaddini, P. C. Hagan, R. Mitra, and B. K. Hebblewhite, "Scale effect on the shear behaviour of rock joints based on a numerical study," Engineering Geology, vol. 181, pp. 212-223, 2014.

[15] J. P. Yang, W. Z. Chen, Y. H. Dai, and H. D. Yu, "Numerical determination of elastic compliance tensor of fractured rock masses by finite element modeling," International Journal of Rock Mechanics and Mining Sciences, vol. 70, pp. 474-482, 2014.

[16] B. A. Poulsen, D. P. Adhikary, M. K. Elmouttie, and A. Wilkins, "Convergence of synthetic rock mass modelling and the Hoek-Brown strength criterion," International Journal of Rock Mechanics and Mining Sciences, vol. 80, pp. 171$180,2015$.

[17] P. Wang, T. Yang, T. Xu, M. Cai, and C. Li, "Numerical analysis on scale effect of elasticity, strength and failure patterns of jointed rock masses," Geosciences Journal, vol. 20, no. 4, pp. 539-549, 2016.

[18] Q. Wu and P. H. S. W. Kulatilake, "REV and its properties on fracture system and mechanical properties, and an orthotropic constitutive model for a jointed rock mass in a dam site in China," Computers and Geotechnics, vol. 43, no. 3, pp. 124142, 2012.

[19] S. Hu, Y. Tan, H. Zhou et al., "Anisotropic modeling of layered rocks incorporating planes of weakness and volumetric stress," Energy Science and Engineering, vol. 8, no. 3, pp. 789-803, 2020.

[20] S. P. Neuman, "Stochastic continuum representation of fractured rock permeability as an alternative to the RVE and fracture network concepts," in Proceedings of the 28th US Symposium of Rock Mechanics, Tucson, America, July 1987.

[21] F. D. Cuisiat and B. C. Haimson, "Scale effects in rock mass stress measurements," International Journal of Rock Mechanics and Mining Sciences, vol. 29, no. 2, pp. 99-117, 1992.

[22] H. Lin, H. Yang, Y. Wang, Y. Zhao, and R. Cao, "Determination of the stress field and crack initiation angle of an open flaw tip under uniaxial compression," Theoretical and Applied Fracture Mechanics, vol. 104, article 102358, 2019.

[23] Y. F. Chen and H. Lin, "Consistency analysis of Hoek-Brown and equivalent Mohr-Coulomb parameters in calculating slope safety factor," Bulletin of Engineering Geology and the Environment, vol. 78, no. 6, pp. 4349-4361, 2019.

[24] T. Zhigang, Z. Chun, H. Manchao, and L. Kuiming, "Research on the safe mining depth of anti-dip bedding slope in Changshanhao Mine," Geomechanics and Geophysics for Geo-Energy and Geo-Resources, vol. 6, no. 2, article 36, 2020.

[25] Z. Z. Liang, Y. B. Zhang, S. B. Tang, L. C. Li, and C. A. Tang, "Size effect of rock messes and associated representative element properties," Chinese Journal of Rock Mechanics and Engineering, vol. 38, pp. 1533-1541, 2013.

[26] Z. Liang, N. Wu, Y. Li, H. Li, and W. Li, "Numerical study on anisotropy of the representative elementary volume of strength and deformability of jointed rock masses," Rock Mechanics and Rock Engineering, vol. 52, no. 11, pp. 4387-4402, 2019.
[27] K. Esmaieli, J. Hadjigeorgiou, and M. Grenon, "Estimating geometrical and mechanical REV based on synthetic rock mass models at Brunswick mine," International Journal of Rock Mechanics and Mining Sciences, vol. 47, no. 6, pp. 915926, 2010.

[28] M. Huang, C. C. Xia, C. R. Ma, H. Zhang, Z. Y. Luo, and C. S. $\mathrm{Xu}$, "A progressive coverage statistical method for series scales three-dimensional morphology of rock joints," Chinese Journal of Rock Mechanics and Engineering, vol. 38, pp. 15331541, 2019.

[29] M. Huang, Z. Y. Luo, and S. G. Du, "Experimental study of sampling representativeness of structural plane of rock model," Chinese Journal of Rock Mechanics and Engineering., vol. 32, no. 10, pp. 2008-2014, 2013.

[30] W. Zhao and C. A. Tang, "The measure theory of discontinuities spacing and trace length," China Mining Magazine, vol. 7, no. 3, pp. 36-38, 1998. 